\title{
Informe Anual del Centro de
}

Derechos Humanos 



\section{Informe de Actividades 2005}

Centro de Derechos Humanos

Facultad de Derecho

Universidad de Chile

\section{DOCENCIA}

El Centro de Derechos Humanos (CDH) ha asumido como uno de sus objetivos centrales la labor docente. Cumpliendo con este fin, el CDH dicta diversos cursos en materia de Derechos Humanos, tanto dentro de la Facultad de Derecho como en actividades docentes de extensión.

\section{DOCENCIA EN PREGRADO}

Como parte del proceso de adecuación y modernización de los planes de estudio de nuestra Facultad de Derecho, se ha establecido, en forma permanente, un Curso Optativo de Derecho Internacional de Ios Derechos Humanos para alumnos que hayan aprobado el ramo de Derecho Internacional Público. Para dicho curso, los co-directores del $\mathrm{CDH}$ han elaborado un programa general de estudios y materiales especiales para el apoyo de la cátedra. Este curso, que recibe también numerosos alumnos extranjeros de programas de intercambio, se imparte en ambos semestres alternadamente por los co-directores del Centro.

Durante el año 2005, la cátedra del primer semestre fue impartida en conjunto por los profesores Cecilia Medina y Claudio Nash. En el segundo semestre de este año la cátedra fue dictada por el profesor José Zalaquett.

\section{DOCENCIA EN POSTGRADO}

El Centro de Derechos Humanos colabora en seminarios y clases sobre derechos humanos en programas de estudios organizados por nuestra Universidad.

\section{PROGRAMA DE DIPLOMAS DE POSTÍTULO}

Una iniciativa que el CDH está llevando a cabo desde el año 2004 es la realización de programas de estudio a nivel de postgrado. Se imparten dos cursos de especialización en derechos humanos: uno de ellos se centra en el estudio de los procesos de democratización y el otro en los derechos humanos de las mujeres.

El objetivo principal de estos cursos es capacitar a profesionales de la región mediante un sistema de educación semipresencial. Esto les permite continuar con sus actividades laborales y al mismo tiempo adquirir nuevas herramientas de trabajo. Así, dentro de un periodo de aproximadamente 
6 meses, los alumnos realizan clases en línea y asisten a tres semanas de clases en Santiago.

Durante los dos años en que estos cursos se han llevado a cabo, el CDH ha recibido alrededor de 180 postulaciones para cada uno, y más de 200 solicitudes de información de diversos países de Latinoamérica.

\section{Diploma de Postítulo "Derechos Humanos y Procesos de Democratización"}

Este diploma de postítulo, organizado en conjunto con el International Center for Transitional Justice (ICTJ) desde el año 2004, ofrece la oportunidad de estudiar temas relacionados con procesos de transición a la democracia y derechos humanos en sociedades afectadas, en el pasado reciente, por regímenes represivos o conflictos armados, así como temas vinculados con el desafío más general de construir sistemas democráticos viables.

Este curso está dirigido a profesionales de diversas disciplinas (derecho, periodismo, educación, trabajo social, entre otras), cuyas actividades actuales demuestran su capacidad de defender y promover los derechos humanos o de influir en la construcción de sociedades democráticas más justas, abiertas y pacíficas en sus países de origen.

En el mes de abril, luego de tres semanas de trabajo de homogeneización en línea, los alumnos asistieron en Chile a dos semanas de clases presenciales donde pudieron compartir con connotadas personalidades nacionales e internacionales como el ex Presidente de la República Patricio Aylwin, Sofía Macher de Perú y Juan Méndez de Argentina; y en el mes de septiembre se llevó a cabo la tercera semana presencial con la cual finalizó este curso. El programa fue calificado por los alumnos como excelente y superior a lo que esperaban.

La tercera versión de este curso comenzará el 13 de marzo de 2006.

\section{Diploma de Postítulo "Derechos Humanos y Mujeres: Teoría y Práctica"}

Este diploma de postítulo tiene como objetivo principal entrenar a abogadas/os de América Latina en el uso de tratados internacionales de derechos humanos, tanto en el ámbito nacional como internacional, para efectos de contribuir al proceso de eliminación de la discriminación contra la mujer. El curso provee conocimientos en Derecho Internacional de los Derechos Humanos y en Teoría Feminista y Género, y destrezas en técnicas de litigación.

La segunda versión de este curso se llevó a cabo entre agosto de 2005 y enero de 2006. Luego de tres semanas de homogeneización en línea, se realizaron las clases presenciales durante la última semana de agosto y la primera de septiembre, donde los alumnos asistieron a charlas de representantes del ACNUR, UNIFEM, el Comité Internacional de la Cruz Roja y UNICEF. La última semana presencial se realizó durante el mes de enero de 2006, instancia en que los alumnos tuvieron la posibilidad 
de debatir distintos temas con interesantes expositores como Francisco Cox (CEJIL), Jessica Neuwirth (Equality Now) y Cecilia Pérez (Ministra del SERNAM).

La tercera versión de este curso comenzará el 31 de julio de 2006.

\section{PROGRAMAS DE MAGÍSTER}

El profesor José Zalaquett dicta el curso obligatorio de Ética y Gobierno en el Magíster de Gestión y Políticas Públicas de la Escuela de Ingeniería Industrial de la Facultad de Ciencias Físicas y Matemáticas de la Universidad de Chile, programa en el que es asistido por el profesor Claudio Nash.

El profesor Claudio Nash dirigió el Seminario sobre "Responsabilidad Internacional de los Estados y Derechos Humanos" para el Programa de Magíster de la Escuela de Graduados de la Facultad de Derecho de la Universidad de Chile (noviembre).

\section{PARTICIPACIÓN EN DIPLOMADO “ESTADO DE DERECHO Y ACCESO A LA JUSTICIA"}

Los profesores Cecilia Medina, José Zalaquett y Claudio Nash, se desempeñaron como docentes en el Diplomado "Estado de Derecho y Acceso a la Justicia", organizado en conjunto por el Centro de Estudios de la Justicia de la Facultad de Derecho, el Heidelberg Center, California School of Law y la GTZ. Este programa se llevó a cabo entre los meses de abril y agosto de 2005.

\section{COMITÉ ACADÉMICO DOCTORADO EN DERECHO}

La profesora Cecilia Medina es miembro del Comité Académico del Doctorado en Derecho ofrecido por la Facultad de Derecho de la Universidad de Chile.

\section{CONSEJO ESCUELA DE GRADUADOS}

El profesor José Zalaquett es miembro del Consejo Escuela de Graduados de la Facultad de Derecho de nuestra Universidad.

\section{DOCENCIA EXTERNA}

Los docentes del Centro de Derechos Humanos han participado en los siguientes cursos o seminarios externos a nuestra Facultad.

Durante este año la profesora Cecilia Medina ha participado, entre otras, en las siguientes actividades:

- Curso El cumplimiento de las sentencias de la Corte Interamericana de Derechos Humanos y Las opiniones consultivas, organizado por el Instituto Interamericano de Derechos Humanos, la Corte 
Interamericana y la Comisión Interamericana para funcionarios públicos (12 de marzo).

- Charla sobre Derecho interno y Derecho internacional, dirigida a jueces de la Corte Suprema, en Asunción, Paraguay (12 de mayo).

- Charla sobre Discriminación y mujeres en el marco de los sistemas de protección de los derechos humanos de las NU y de la OEA, a alumnos y docentes de la Facultad de Derecho, Universidad Católica, Asunción, Paraguay (12 de mayo).

- Programa de las Naciones Unidas para el Desarrollo (SURF-LAC) y Oficina del Alto Comisionado de las Naciones Unidas para los Derechos Humanos (Oficina Regional-LAC): Taller interagencial sobre recomendaciones de órganos de tratados y programación basada en derechos. Clase sobre Evolución e impacto jurídico de las recomendaciones de los órganos de tratados a nivel nacional, Santiago, Chile (17 de mayo).

- Participación en el Comité de Selección de Becas Ford para personas de grupos vulnerables (19-20 y 30 de mayo).

- Participación en coloquio Judicial sobre la Aplicación de Normas Internacionales de Derechos Humanos en el Plano Interno, en apoyo de la implementación de la Convención para la Eliminación de toda forma de Discriminación contra la Mujer, organizado por la División para el Avance de la Mujer (DAW) de las Naciones Unidas y la Comisión Económica para América Latina y el Caribe (CEPAL). Facilitadora de talleres de trabajo (25-27 de mayo).

- Charla sobre Obligación de los Estados de garantizar los derechos políticos de las mujeres en Seminario sobre "Democracia y Paridad", organizado por Humanas, Instituto de la Mujer, OXFAM. Actividad auspiciada por el British Council y la Embajada de Francia (30 de septiembre).

- Participación en Mesa Redonda sobre los Principios de París y los proyectos de ley sobre Defensor del Ciudadano y el Instituto Nacional de Derechos Humanos, organizada por el Ministerio de Relaciones Exteriores de Chile (3 de octubre).

- Charla sobre Derecho Internacional de los Derechos Humanos y Derechos de las Mujeres en el Curso especializado sobre derechos de las Mujeres organizado por el Instituto Interamericano de Derechos Humanos, San José Costa Rica (23 de noviembre).

- Participación en panel sobre Derechos Económicos, Sociales y Culturales y la jurisprudencia de la Corte Interamericana de Derechos Humanos, organizado por la Universidad Diego Portales (6 de diciembre). 
El profesor José Zalaquett ha participado, entre otras, en las siguientes actividades académicas externas a la Facultad:

- Conferencia sobre "Ética Política" en la jornada anual de los académicos de la Facultad de Economía de la Universidad Católica de Chile (12 de enero).

- Conferencia de inauguración del año académico de la Facultad de Filosofía y Humanidades, Universidad de Chile (6 de abril).

- Conferencia de inauguración del año académico, Escuela de Derecho, Universidad de Valparaíso (27 de abril).

- Taller sobre Derechos Humanos para una delegación de jueces de Haití (2 de mayo).

- Taller sobre transición a la democracia para una delegación visitante de abogados de países árabes (3 de mayo).

- Participación en el jurado de selección de becas de postgrado para postulantes de Perú y Chile, programa de la Fundación Ford (4-5 de julio).

- Conferencia sobre Desobediencia Civil y Terrorismo en el II Congreso Estudiantil de Derecho y Teoría Constitucional, Facultad de Derecho, Universidad de Chile (12 de agosto).

- Conferencia La Desobediencia civil en Rawls y la Ética de las Medidas Excepcionales y Medidas Extremas, dictada en el encuentro sobre El pensamiento filosófico, político y jurídico de John Rawls, Universidad de Valparaíso (2 de septiembre).

- Charla en Seminario Internacional Promoviendo una cultura de transparencia a través del Acceso a la Información Pública: Buenas Prácticas en Chile y el mundo, organizado por Proacceso (4 de octubre).

- Participación en conferencia sobre Nuevas tendencias en el Derecho Internacional, organizada por la Agencia de Cooperación Alemana GTZ y el Programa de Naciones Unidas para el Desarrollo, Berlín, Alemania (21 y 22 de noviembre).

- Participación en conferencia sobre La reforma a la Justicia en las Américas, Montevideo, Uruguay, organizada por el Instituto de Estudios Legales y Sociales del Uruguay (1 y 2 de diciembre).

El profesor Claudio Nash ha participado en las siguientes actividades externas a la Universidad:

- Participación en Seminario SELA, sobre "Derecho y Pobreza", organizado por la Facultad de Derecho de la Universidad de Yale, Río de Janeiro, Brasil (16-19 de junio).

- Ponencia "Las reparaciones por violaciones a los derechos humanos", y clase "Trabajo con casos de reparaciones", en el seminario "Las reparaciones del daño por violación de los derechos humanos", 
organizado por la Universidad Iberamericana de México y por la Comisión de Derechos Humanos del Distrito Federal (4 y 5 de julio).

- Docente en el Curso "Visión de Chile Contemporáneo", organizado por el Programa de Movilidad Estudiantil - Universidad de Chile, dirigido a alumnos de intercambio (julio).

- Charla sobre "Los derechos indígenas en el sistema interamericano de derechos humanos" en el Diplomado "Derechos Indígenas y Derechos Humanos" organizado por la Facultad de Derecho de la Universidad Católica de Temuco y el Observatorio de Derechos Indígenas (10 de diciembre).

Dentro de la labor de docencia externa que realiza en $\mathrm{CDH}$, especial importancia revisten aquellas realizadas en coordinación con otras instituciones.

En el marco del Programa de Formación de Defensores Públicos, organizado por la Defensoría Penal Pública, Cecilia Medina y Claudio Nash realizaron el módulo "Derechos Humanos y Debido Proceso" (21-24 de marzo).

\section{PROYECTOS ESPECIALES E INVESTIGACIÓN}

Durante el año 2005 el Centro de Derechos Humanos ha realizado labores de investigación y asesoría en los siguientes proyectos:

\section{- Proyecto sobre Rendición de Cuentas, Lucha contra la Corrupción y Transparencia.}

Entendiendo el fortalecimiento de la democracia como un área de especial relevancia en materia de derechos humanos, el $\mathrm{CDH}$ está Ilevando a cabo un programa centrado en temas de transparencia, accountability y lucha contra la corrupción. En esta actividad contamos con el apoyo de The Open Society Institute, quien financia y co-organiza este proyecto.

Para comenzar el trabajo en esta área se Ilevó a cabo una conferencia de consulta y debate en diciembre de 2004, cuyos objetivos, entre otros, fueron: la evaluación de la situación actual en materia de anticorrupción en Latinoamérica y el mundo; la evaluación de los recursos existentes para ser empleados en la lucha contra la corrupción; el fortalecimiento de redes; la clarificación de conceptos relevantes; el establecimiento de prioridades para el trabajo futuro, y la discusión de estrategias para el avance hacia objetivos comunes.

A esta reunión, denominada "Reunión Regional sobre Transparencia, Rendición de Cuentas y Lucha contra la Corrupción en América", asistieron veintisiete expertos en materias relacionadas con la transparencia, provenientes de destacadas ONGs, instituciones donantes, establecimientos de educación superior y organismos internacionales de América y Europa. 
En octubre de 2005 el CDH publicó el informe final de esta reunión elaborado por José Zalaquett, donde se presentan las ideas que surgieron del debate (ver sección Publicaciones).

\section{- Proyecto "Los Derechos Humanos consagrados por la Convención} Americana sobre Derechos Humanos a la luz, principalmente, de la jurisprudencia de la Corte Interamericana de Derechos Humanos".

Este proyecto, realizado entre el año 2002 y 2005, dirigido por la profesora Cecilia Medina Quiroga y asistido por Claudio Nash Rojas, cuenta con el financiamiento de la Fundación Ford. Actualmente se encuentra en la etapa final de trabajo.

El resultado de la investigación se tradujo en la publicación del libro La Convención Americana: Teoría y Jurisprudencia, de Cecilia Medina en junio de 2005 (ver más adelante). En conjunto con este libro, se publicó un subproducto de investigación relativo a las Reparaciones en la Jurisprudencia de la Corte Interamericana de Derechos Humanos, elaborado por el profesor Claudio Nash y publicado en octubre de 2004. En el año 2006, se presentará la tercera publicación de este proyecto.

\section{- Publicaciones Diploma de Postítulo "Derechos Humanos y Mujeres: Teoría y Práctica".}

Asociado a este programa de estudios se publicó una recopilación de la interpretación oficial de los tratados internacionales por los organismos monitores de su cumplimiento, en diciembre de 2005 (ver sección publicaciones); y se publicará otra recopilación de la jurisprudencia internacional en materia de género, en el año 2006.

\section{ANUARIO DE DERECHOS HUMANOS}

En agosto de este año se presentó el Anuario de Derechos Humanos elaborado por el CDH con el fin de contribuir a la difusión, promoción, enseñanza y debate sobre derechos humanos.

Esta publicación se concibe como un libro de referencia y consulta sobre materias de derechos humanos y campos afines. Pretende poner a disposición del público los distintos desarrollos en materia de derechos humanos, tanto a nivel nacional como internacional, dentro del período anual cubierto por cada edición (el año anterior al de su publicación).

Su contenido comprende artículos doctrinarios -la primera versión contó con artículos de Ronald Dworkin y Michael Ignatieff-y de actualidad en derechos humanos, análisis jurisprudenciales y normativos, tanto a nivel nacional como internacional. A su vez, cada edición tiene una sección especial destinada a la exposición de materias afines al campo de los derechos humanos. Este año se abordó el tema de transparencia, rendición de cuentas y lucha contra la corrupción. 
Con el fin de dar la mayor difusión posible a esta nueva fuente debate el $\mathrm{CDH}$, con el apoyo de SISIB de la Universidad de Chile, elaboró una página web para dar soporte electrónico a esta publicación: www.anuariocdh.uchile.cl, la cual fue presentada en la misma oportunidad que el Anuario.

Conjuntamente con esta página, se lanzó el sitio www.publicacionescdh.uchile.cl, en el que están disponibles las publicaciones del $\mathrm{CDH}$.

\section{DIRECCIÓN DE MEMORIAS Y TESIS}

Uno de los objetivos del Centro de Derechos Humanos es el fomento de la investigación en materia de derechos humanos; en ese entendido es preocupación de los co-directores colaborar en la ejecución de memorias y tesis sobre temas relevantes vinculados a las materias de interés para el Centro. Actualmente ambos co-directores dirigen a estudiantes en la elaboración de sus memorias para optar al grado de Licenciado en Ciencias Jurídicas y Sociales.

Durante este año se ha guiado las siguientes memorias de grado:

- Memoria del alumno Franz Möller, Las soluciones amistosas en las peticiones individuales del sistema interamericano de promoción y protección de los derechos humanos, dirigida por Cecilia Medina.

- Memoria del alumno Felipe González San Martín, La teoría de la guerra justa a la luz de los nuevos desafíos en materia de seguridad internacional y derechos humanos, dirigida por José Zalaquett.

- Memoria de las alumnas Valentina Monasterio y Ana María Echeverría, "Instituto Nacional de Derechos Humanos: el proyecto chileno", dirigida por José Zalaquett.

- Memoria del alumno Cristián Fernández, "Algunos problemas en la implementación de la Corte Penal Internacional de su Estatuto y la necesidad de su funcionamiento", dirigida por Cecilia Medina.

\section{CÍRCULO DE ESTUDIOS EN DERECHOS HUMANOS}

Una de las actividades que el CDH realiza desde el año 2002 es el Círculo de Estudios en Derechos Humanos, instancia de debate y conversación sobre diversos temas, nacionales e internacionales, en materia de derechos humanos, cuya organización y desarrollo están a cargo de José Zalaquett y María José Eva. Actualmente el grupo está conformado por aproximadamente 30 profesionales y académicos.

Durante el primer semestre de este año las reuniones versaron sobre Derechos Humanos y Derecho de Autor, y el estudio de un artículo de Michael Ignatieff titulado "la Democracia y el Mal Menor".

Las actividades del Círculo de Estudios serán retomadas en marzo de 2006 con una nueva forma de trabajo y presentación de temas. 


\section{TALLER DE ENSEÑANZA EN DERECHOS HUMANOS}

Dado que entre los objetivos institucionales del $\mathrm{CDH}$ se considera el contribuir al perfeccionamiento de estudiantes de derecho con miras, entre otras cosas, a prepararlos para participar en los concursos internacionales sobre la materia que se realizan anualmente, se ha invitado a un grupo de ellos a conformar el Taller de Enseñanza en Derechos Humanos. Este taller, que se creó el segundo semestre de 2003, se desarrolla dos veces al mes convocando a estudiantes a participar en el debate, crítica y estudio de jurisprudencia internacional en materia de derechos humanos.

Este año el Taller, que está a cargo de Cecilia Medina y Patricia Palacios, convocó a 20 estudiantes de la Facultad y se llevó a cabo durante el segundo semestre. En él se abordaron los siguientes temas: el derecho a la vida, la integridad personal y el debido proceso.

\section{COMPETENCIAS INTERNACIONALES}

Como parte del proceso de formación y capacitación en materia de derechos humanos, dentro del marco de actividades que el $\mathrm{CDH}$ realiza, está la preparación de alumnos para participar en distintas competencias internacionales sobre derechos humanos.

Durante el segundo semestre de este año, el CDH seleccionó a los dos alumnos que representarán a la Facultad de Derecho en el XI Concurso Interamericano de Derechos Humanos organizado por la American University que se llevará a cabo en mayo de 2006: Valeska David y Alia Trabucco.

\section{EVENTOS ACADÉMICOS}

\section{SEMINARIO EN LATINOAMÉRICA DE TEORÍA CONSTITUCIONAL Y POLÍTICA (SELA)}

SELA es un encuentro anual que reúne a académicos y estudiantes de Argentina, Brasil, Chile, Colombia, México, Paraguay, Perú, Puerto Rico, España y Estados Unidos, particularmente de la Universidad de Yale. Cada año se trata un tema distinto y se presentan papers dirigidos a formular un debate entre los asistentes.

Este año el Centro de Derechos Humanos coordinó la participación de los académicos de la Facultad de Derecho de la Universidad de Chile en este seminario llevado a cabo los primeros días de junio en Río de Janeiro, Brasil.

\section{CHARLA SOBRE TRANSPARENCIA Y LUCHA CONTRA LA CORRUPCION}

Con motivo de la presencia del señor José Ugaz, Senior Institutional Integrity Officer del Banco Mundial, el CDH organizó la conferencia Políticas del Banco Mundial sobre Probidad Pública, Transparencia y Combate a la Corrupción. 
Junto con esta actividad, que tuvo lugar el 4 de octubre, se llevó a cabo la presentación del libro Transparencia, rendición de cuentas y lucha contra la corrupción en América, editado por el Centro de Derechos Humanos.

El año 2005 el Centro de Derechos Humanos ha creado la página web www.publicacionescdh.uchile.cl, con el objeto de dar la mayor difusión posible al trabajo de investigación que lleva a cabo. En este sitio es posible encontrar artículos y conferencias realizadas por los miembros del Centro, así como también, información relacionada con los cursos a distancia que desarrolla.

Además, conjunto con esta página, se creó un sitio que acoge el Anuario de Derechos Humanos, donde es factible encontrar, además de los artículos en él publicados, los fallos, leyes y documentos a los cuales se hace referencia en cada uno de ellos. 